\title{
Ruptura esplénica espontánea en paciente con litiasis renal derecha
}

\author{
Cynthia G. Castro-Caballero ${ }^{1}$ y Jesús A. Aco-Luna2*
}

${ }^{1}$ Servicio de Urgencias; ${ }^{2}$ Servicio de la especialidad de Urgencias Médico-Quirúrgicas. Hospital General Regional N. ${ }^{1}$, Instituto Mexicano del Seguro Social, Orizaba, Veracruz, México

\begin{abstract}
Resumen
El ingreso al área de reanimación de pacientes con estado de choque nos lleva a indagar e identificar el mecanismo primario de su desarrollo. La evaluación primaria nos brinda datos importantes sobre su condición, y un estado de hipoperfusión nos obliga a implementar tratamientos precisos. Se trata el caso de una mujer de 65 años con choque séptico por litiasis ureteral complicado con absceso renal que ameritó tratamiento quirúrgico y antimicrobiano. Tras presentar súbita inestabilidad hemodinámica y sospecha de nuevo absceso renal se ingresó a quirófano, donde se realizó nefrectomía izquierda. Durante el transoperatorio se evidenció hemoperitoneo secundario a ruptura total de bazo y se procedió a esplenectomía. No se documentó trauma o uso de anticoagulantes previos. La ruptura esplénica atraumática es una patología poco frecuente y usualmente subestimada, pero que se debe tener como sospecha diagnóstica en el paciente con estado de choque hipovolémico hemorrágico atraumático. Es de suma importancia el pronto reconocimiento para evitar complicaciones y mejorar la supervivencia.
\end{abstract}

Palabras clave: Choque. Ruptura esplénica espontanea. Absceso renal. Reporte de caso.

\section{Spontaneous splenic rupture in a patient with right kidney stones}

\section{Abstract}

The patient's arrival to the resuscitation area with a shock state take us to inquire and identify the major mechanism of its development. The primary assessment provides us significant information about its condition, and a hypoperfusion state force us to implement accurate treatment strategies. A 65 years old woman presented with septic shock due to ureteral lithiasis complicated with renal abscess that required surgery and antibiotic treatment. Clinically the patient's condition deteriorated with sudden hemodynamic instability, a renal abscess was suspected and left nephrectomy was performed. During transoperatory was found hemoperitoneum due to spontaneous splenic rupture and splenectomy was performed., No prior trauma or anticoagulation drug therapy was documented. The non-traumatic splenic rupture is an unusual and occasionally underrated, but must be suspected in a non-traumatic shock state. It is important the early recognize to avoid complications and improve survival.

Key words: Shock. Renal abscess. Splenic rupture. Case report.

\section{Correspondencia:}

*Jesús A. Aco-Luna

E-mail: jealaclu@gmail.com
Disponible en internet: 25-11-2021

Fecha de recepción: 16-03-2021

Fecha de aceptación: 28-08-2021 DOI: 10.24875/REIE.21000019
Rev Educ Investig Emer. 2021;3(4):226-229 www.medicinadeemergencias.com

604-6520 (c) 2021 Sociedad Mexicana de Medicina de Emergencias, AC. Publicado por Permanyer México SA de CV. Este es un artículo open access bajo la licencia CC BY-NC-ND (http://creativecommons.org/licenses/by-nc-nd/4.0/). 


\section{Introducción}

El estado de choque se define como un fallo circulatorio asociado a la incapacidad para cumplir una adecuada entrega de oxígeno a nivel tisular. La circulación es inhábil para cumplir las demandas tisulares de oxígeno y el resultado es la disoxia celular ${ }^{1,2}$. Puede ser el resultado de cuatro mecanismos o la combinación de estos: pérdida de volumen circulante (hipovolémico), pérdida de contractilidad (cardiogénico), inadecuada contractilidad por obstrucción (obstructivo) o por pérdida del tono vascular (distributivo) ${ }^{3}$. En diversas ocasiones nos enfrentamos ante una combinación de tipos de choque, pues a menudo tienden a estar superpuestos, por lo que es de vital importancia identificarlos oportunamente, para así dar un ágil diagnóstico e iniciar su tratamiento inmediatamente para evitar mayores complicaciones ${ }^{4,5}$.

Es común encontrar a pacientes con choque séptico que en estadios tardíos desarrollan choque obstructivo secundario a miocardiopatía. Una de las variantes menos frecuentes es la asociación del choque séptico con choque hipovolémico, tal es el caso que a continuación les presentamos. Se presenta el caso de un paciente con choque séptico secundario a uropatía obstructiva con absceso renal que no revierte de manera adecuada ante apoyo vasopresor y antimicrobiano, con súbita inestabilidad hemodinámica y datos de hipovolemia por ruptura esplénica (RS) espontánea, sin estar en relación con un evento traumático o uso de anticoagulantes.

\section{Descripción del caso}

Mujer de 65 años de edad, originaria y residente de Orizaba, Veracruz, con antecedentes de hipertensión arterial sistémica en manejo con losartán $50 \mathrm{mg}$ VO cada 12 horas, diabetes mellitus 2 en manejo con insulina glargina $28 \mathrm{UI}$ preprandial y obesidad. Acudió al servicio de urgencias el día 08.09.2021 por dolor abdominal asociado a litiasis renoureteral derecha, siendo candidata a colocación de catéter de pigtail y drenaje de absceso renal derecho sin complicaciones transquirúrgicas ni postquirúrgicas, por lo cual es egresada tras mejoría con antimicrobianos y analgésicos no esteroideos. La paciente reingresó el día 18.09.2020 tras presentar dolor súbito en región lumbar izquierda con datos de choque (tensión arterial [TA] 90/50 mmHg, frecuencia cardiaca $115 \mathrm{lpm}$, frecuencia respiratoria 26 rpm, saturación de oxígeno 96\%, temperatura $37^{\circ}$ C e índice de choque 1.27, acompañado de somnolencia. A su ingreso se presentó neurológicamente con
FOUR (Full Outline of UnResponsiveness) de 13 (E2/ $M 3 / B 4 / R 4)$, sin datos de irritación meníngea, ni liberación extrapiramidal, palidez franca y generalizada de tegumentos, mucosa oral seca, polipneica, tórax simétrico, con hipoventilación basal derecha, sin agregados, mantuvo oximetría de $96 \%$ al medio ambiente, abdomen globoso a expensas de panículo adiposo, mate a la percusión de ambos flancos, sin visceromegalias palpables, doloroso a palpación de cuadrante superior izquierdo, sonda urinaria permeable con escaso gasto urinario, extremidades íntegras, con edema marcado, llenado capilar de 5 segundos.

Se inició apoyo vasopresor para mantener TA media perfusoria (norepinefrina $8 \mathrm{mcg} / \mathrm{min}$ ), además de manejo antimicrobiano dual empírico tras diagnóstico de choque séptico de etiología urinaria. Se realizó medición de gases arteriales que demostró acidosis metabólica (pH 7.34, presión parcial de dióxido de carbono $21 \mathrm{mmHg}$, presión arterial de oxígeno $112 \mathrm{mmHg}$, exceso de base -14.5 y bicarbonato $11.3 \mathrm{mmol} / \mathrm{l}$ (Tabla 1). Más tarde se realizó tomografía simple abdominal que reveló hidronefrosis severa izquierda por uropatía obstructiva litiásica en uretero-proximal además de líquido libre en cavidad con colección en hipocondrio derecho (Fig. 1).

Inesperadamente presentó pobre respuesta a apoyo aminérgico con deterioro neurológico súbito además de disnea, por lo que se decide manejo avanzado de vía aérea. Recibe valoración por parte del servicio de urología, que determinó ingreso a área de quirófano para realización de nefrectomía izquierda. Durante el periodo transoperatorio se visualizó hemoperitoneo de aproximadamente 2,000 ml, por lo que intervino el servicio de cirugía general para posteriormente realizar laparotomía exploradora con hallazgos de RS y finalmente se procedió a esplenectomía total (Fig. 2). Durante el evento quirúrgico se transfundieron cuatro concentrados globulares y cinco plasmas frescos.

Fue manejada durante nueve días en área de choque y tras soporte ventilatorio durante 50 horas desde su ingreso se logró la retirada del apoyo mecánico ventilatorio sin fallo ventilatorio posterior. Tras recuperación de estado hemodinámico y condiciones generales es egresada a cargo de urología para continuar manejo ambulatorio.

\section{Discusión}

La RS tiene distintos orígenes (hematológicos, tumores primarios del bazo, metástasis esplénicas, enfermedades de almacenamiento, infecciosas, misceláneas, 


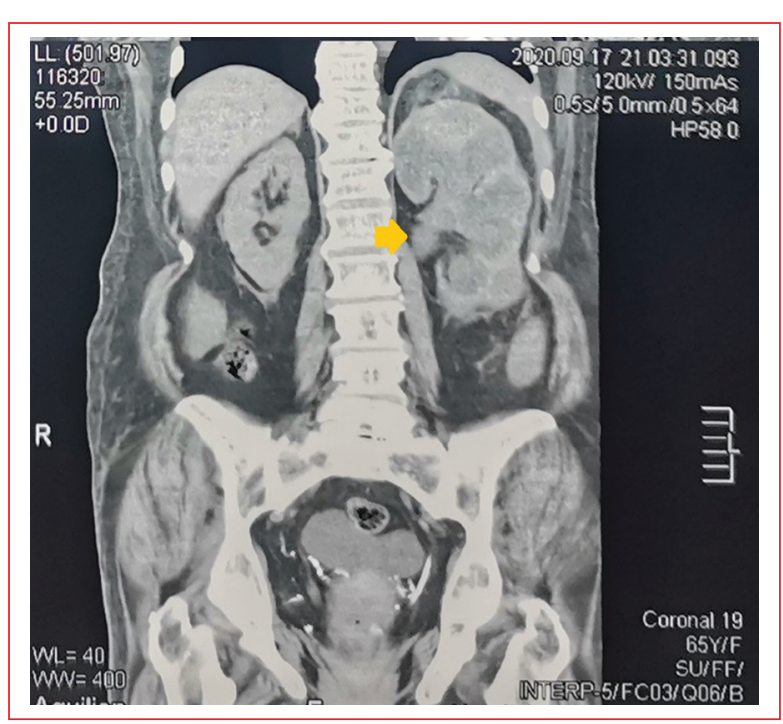

Figura 1. Corte coronal con hidronefrosis por uropatía obstructiva.

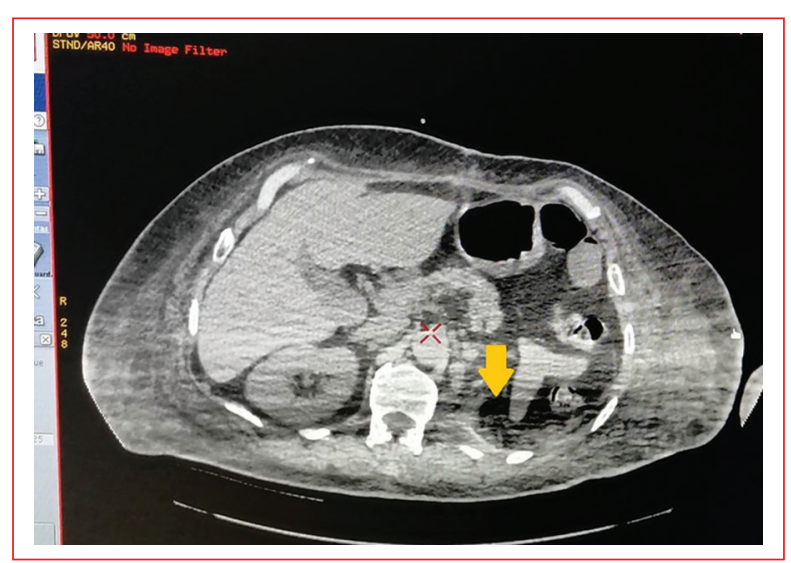

Figura 2. Corte axial posterior a esplenectomía.

iatrogénicas); al no reconocer una lesión histopatológica se clasifica como espontánea ${ }^{6,7}$.

En el mundo existen pocas publicaciones sobre los casos de RS espontánea. Se realizó una búsqueda sobre RS en PubMed con uso de terminología MeSH y Google académico, encontrando dos estudios retrospectivos. En un estudio de revisión se encontraron 613 casos en el periodo de 1950 a $2011^{8}$, en otro se encontraron 845 pacientes durante 1980 a $2008^{9}$.

Se describen tres posibles mecanismos de lesión ${ }^{10}$ :

- Elevación de la tensión intraesplénica, favorecida por la hiperplasia y congestión vascular.

- Compresión brusca ocasionada por la musculatura abdominal o diafragmática, por ejemplo en la maniobra de Valsalva o en casos de emesis violenta.
Tabla 1. Laboratorios

\begin{tabular}{|c|c|c|}
\hline \multicolumn{3}{|c|}{ Laboratorios (18.09.2020) } \\
\hline \multicolumn{2}{|l|}{ 04:35 h } & \multirow{2}{*}{$\begin{array}{l}11: 23 \text { h } \\
\text { pH } 7.18\end{array}$} \\
\hline $\mathrm{Hb} 4.8 \mathrm{~g} / \mathrm{dl}$ & pH 7.34 & \\
\hline Hto $14.4 \%$ & $\mathrm{PCO}_{2} 21 \mathrm{mmHg}$ & $\mathrm{PCO}_{2} 18 \mathrm{mmHg}$ \\
\hline VCM $78.3 \mathrm{fl}$ & $\mathrm{PO}_{2} 112 \mathrm{mmHg}$ & $\mathrm{PO}_{2} 138 \mathrm{mmHg}$ \\
\hline CMHC $33.3 \mathrm{~g} / \mathrm{dl}$ & $\mathrm{HCO}_{3} 14.5 \mathrm{mmol} / \mathrm{l}$ & $\mathrm{HCO}_{3} 6.3 \mathrm{mmol} / \mathrm{l}$ \\
\hline Plts $397.000 \mathrm{mmc}$ & $\mathrm{BE}-14.5 \mathrm{mmol} / \mathrm{l}$ & $\mathrm{BE}-22.1 \mathrm{mmol} / \mathrm{l}$ \\
\hline Leucos $21.230 \mathrm{mmc}$ & $\mathrm{Hb} 4.7 \mathrm{~g} / \mathrm{dl}$ & $\mathrm{Hb} 5.3 \mathrm{~g} / \mathrm{dl}$ \\
\hline Neutros $19.07 \mathrm{mmc}$ & Hto $15 \%$ & Hto $17 \%$ \\
\hline Glucosa $405 \mathrm{mg} / \mathrm{dl}$ & Sat $98 \%$ & Sat $98 \%$ \\
\hline Creat $2.5 \mathrm{mg} / \mathrm{dl}$ & & $\begin{array}{l}\text { Lactato } \\
14.2 \mathrm{mmol} / \mathrm{l}\end{array}$ \\
\hline \multicolumn{3}{|l|}{ K $5.5 \mathrm{mmol} / \mathrm{l}$} \\
\hline $\mathrm{Na} 120 \mathrm{mmol} / \mathrm{l}$ & & \\
\hline
\end{tabular}

$\mathrm{Hb}$ : hemoglobina; Hto: hematocrito; $\mathrm{PCO}_{2}$ : presión parcial de dióxido de carbono; $\mathrm{PO}_{2}$ : presión arterial de oxígeno; VCM: volumen corpuscular medio; $\mathrm{CMHC}$ : concentración de hemoglobina corpuscular media; $\mathrm{HCO}_{3}$ : bicarbonato; Plts: plaquetas; $\mathrm{BE}$ exceso de base Sat: saturación; Creat: creatinina; K: potasio; Na: sodio.

- Oclusión vascular. La hiperplasia reticuloendotelial favorece la formación de trombosis, el desarrollo de infarto y, en último caso, la rotura.

Los métodos de imagen son de apoyo diagnóstico en el caso de una hemorragia intraabdominal, y deben realizarse de manera rápida para el tratamiento del paciente ${ }^{11}$. Los más aceptados como apoyo diagnóstico son el ultrasonido en el punto de atención del paciente ${ }^{12,13}$ y la tomografía computarizada simple de abdomen $^{10}$, y para el diagnóstico final es la laparotomía ${ }^{9}$.

En el caso presentado anteriormente no se cuenta con un estudio histopatológico, pero al no asociarse a trauma y no conocer historia previa de tumoración se clasifica como espontánea, sin embargo se debe indagar la causa para su tratamiento ${ }^{6}$.

Como parte de la pandemia ocasionada por la COVID-19 (enfermedad por coronavirus 2019) las unidades de cuidados intensivos se han reconvertido para la atención de pacientes con patologías respiratorias, por lo que en nuestra unidad la atención de pacientes graves ha regresado al área de choque.

\section{Conclusión}

La RS es un padecimiento muy poco diagnosticado en nuestro medio que no debe ser subestimado, ya que su tratamiento debe ser inmediato. Debemos 
apoyarnos en estudios paraclínicos que nos orienten en su diagnóstico.

\section{Agradecimientos}

Se agradece al personal del Hospital General Regional N. 1 de Orizaba, Veracruz, Lic. Ignacio García Téllez. Agradecemos al personal del Servicio de Urgencias del Hospital General Regional de Orizaba.

\section{Financiamiento}

Los autores declaran no haber recibido ningún tipo de financiamiento.

\section{Conflicto de intereses}

No se declara algún conflicto de intereses por parte de los autores.

\section{Responsabilidades éticas}

Protección de personas y animales. Los autores declaran que para esta investigación no se han realizado experimentos en seres humanos ni en animales.

Confidencialidad de los datos. Los autores declaran que han seguido los protocolos de su centro de trabajo sobre la publicación de datos de pacientes.

Derecho a la privacidad y consentimiento informado. Los autores han obtenido el consentimiento informado de los pacientes y/o sujetos referidos en el artículo. Este documento obra en poder del autor de correspondencia.

\section{Bibliografía}

1. Cecconi M, De Backer D, Antonelli M, Beale R, Bakker J, Hofer C, et al. Consensus on circulatory shock and hemodynamic monitoring. Task force of the European Society of Intensive Care Medicine. Intensive Care Med. 2014;40(12):1795-815

2. Lee MJ. Non-traumatic abdominal emergencies: imaging and intervention in sepsis. Eur Radiol. 2002;12(9):2172-9.

3. Kislitsina ON, Rich JD, Wilcox JE, Pham DT, Churyla A, Vorovich EB et al. Shock - Classification and pathophysiological principles of therapeutics. Curr Cardiol Rev. 2019;15(2):102-13.

4. Herness J, Buttolph A, Hammer NC. Acute pyelonephritis in adults: Rapid evidence review. Am Fam Physician. 2020;102(3):173-80.

5. Medina-Escobedo M, Alcocer-Dzul R, López-LópezJ, Villanueva-JorgeS. Obesidad como factor de riesgo para alteraciones metabólicas en adultos con litiasis urinaria. Rev Med Inst Mex Seguro Soc. 2015; 53(6):692-7.

6. Susaeta R, Benavente D, Marchant F, Gana R. Diagnóstico y manejo de litiasis renales en adultos y niños. Rev Med Clínica Las Condes. 2018;29(2):197-212.

7. López-Tomassetti Fernández EM, Delgado Plasencia L, Arteaga González IJ, Carrillo Pallares Á, Hernández Siverio N. Rotura no traumática del bazo: experiencia con 10 casos. Gastroenterol Hepatol. 2007;30(10):585-91.

8. Gómez-Ramos JJ, Marín-Medina A, Lisjuan-Bracamontes J, García-Ramírez D, Gust-Parra H, Ascencio-Rodríguez MG. Adolescent with spontaneous splenic rupture as a cause of hemoperitoneum in the Emergency Department: Case report and literature review. Pediatr Emerg Care. 2020;36(12):e737-41.

9. Aubrey-Bassler FK, Sowers N. 613 cases of splenic rupture without risk factors or previously diagnosed disease: a systematic review. BMC Emerg Med. 2012;12:11.

10. Guantes del Vigo MB, Hernaiz Argudo L, Insausti Jaca N, Larrazabal Echevarría E, Aperribay Ulacia M, Casadiego Matarranz L. Rotura esplénica atraumática: revisión [Internet]. Seram, Sociedad Española de Radiología Médica; 2018. Disponible en: https://piper.espacio-seram. com/index.php/seram/article/view/857/498

11. Renzulli P, Hostettler A, Schoepfer AM, Gloor B, Candinas D. Systematic review of atraumatic splenic rupture. Br J Surg. 2009;96(10):1114-21.

12. Espil G, Larrañaga N, Díaz Villarroel N, Oyarzun A, Matzke G, Kozima S. Hemorragia abdominal espontánea: evaluación por imágenes. Rev Argent Radiol. 2015;79(2):86-94.

13. Whitson MR, Mayo PH. Ultrasonography in the emergency department. Crit Care. 2016;20(1):227. 